CV.nana استخدام بعض منظمات النمو في استحداث وتمايز الكالس لإعادة تكوين نباتات القديفة القزمية

\title{
Tagetes patula
}

r- - تمايز الكالس وتكوين الأفرع الخضرية

قاسم محمود الحمداني

قسم المحاصيل/كلية الزر اعة والغابات/جامعة الموصل

\section{الخلاصة}

أظهرت هذه الدر اسة قابلية كالس نباتات القديفة القزمية T. patula cv.nana على التمايز وتكوين

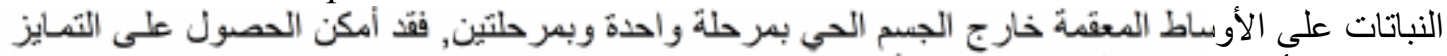

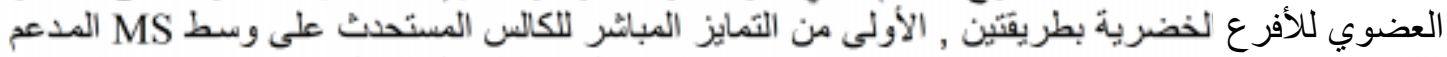

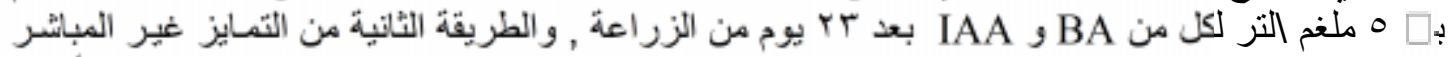

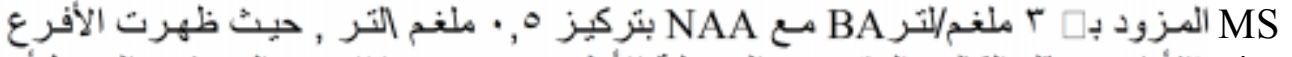

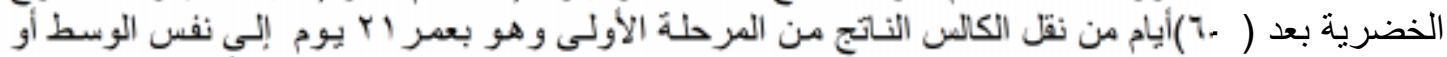

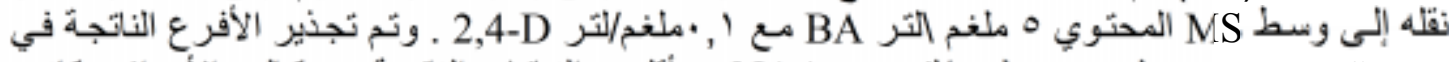

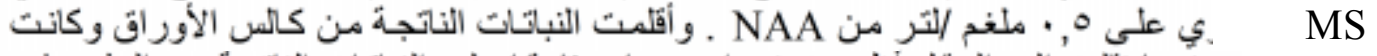

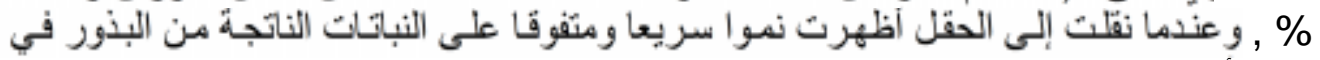

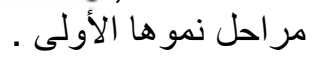

\section{المقدمة}

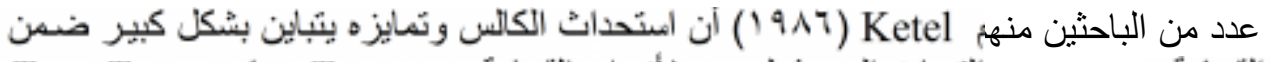

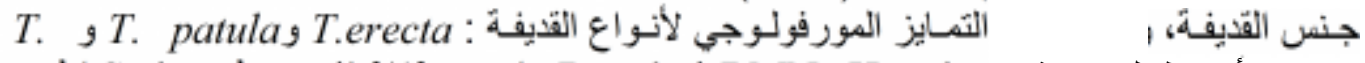
الأوساط الزر اعية Hinuta

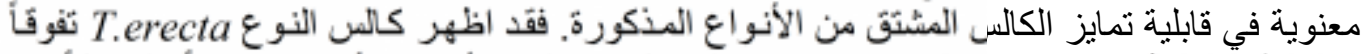

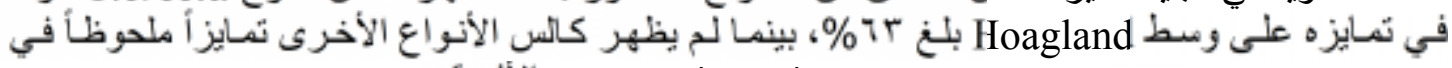

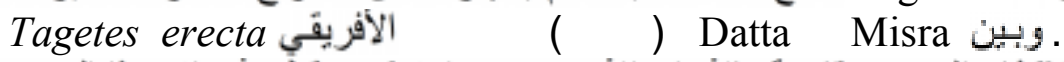

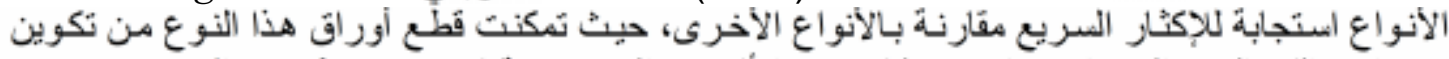

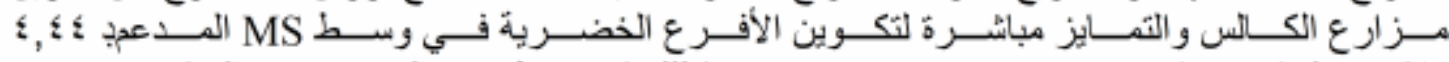

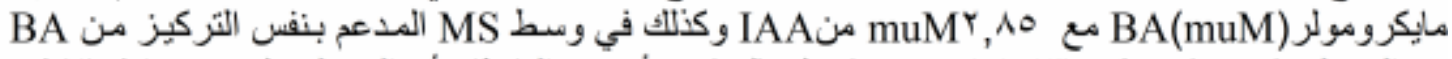

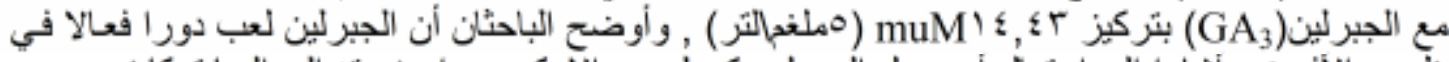

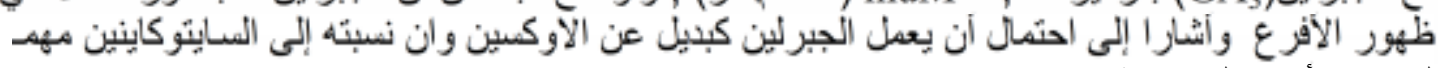

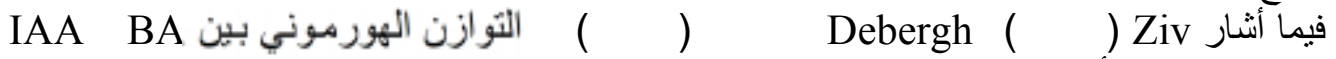
كان مهما لتحفيز نشوء الأفرع وان زيادة تركيز

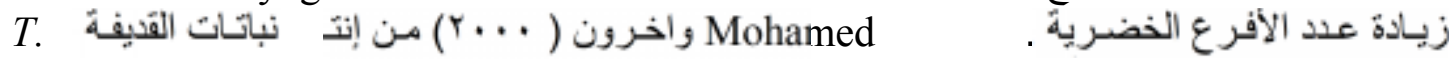

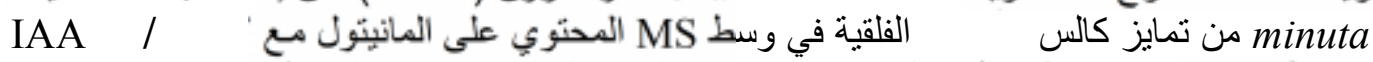

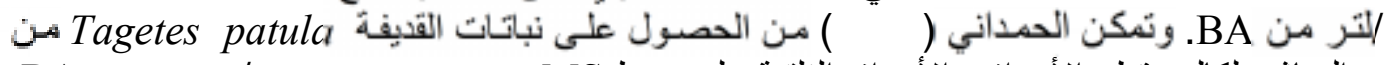

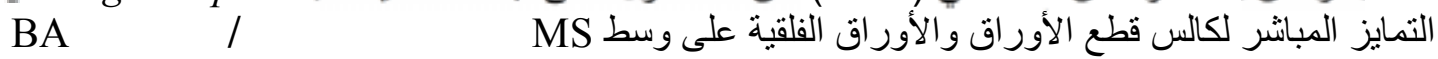

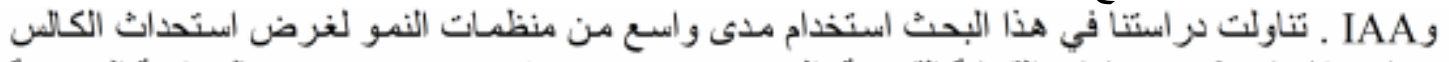

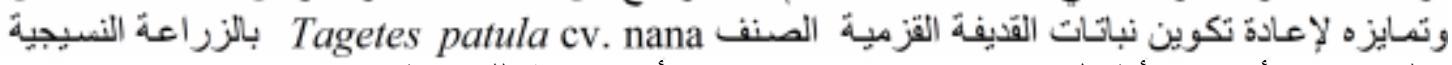
التي تمتاز بأنها ذات أطو ال قصيرة لان تتجاوز سم والأز هار بنية اللون تظهر مبكرة .

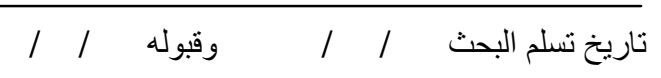

مواد البحث وطر ائقه

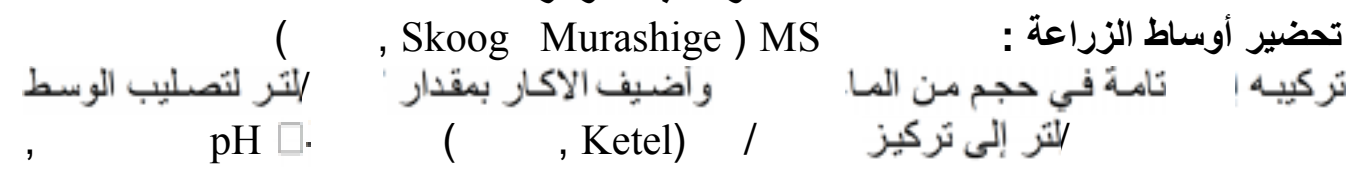




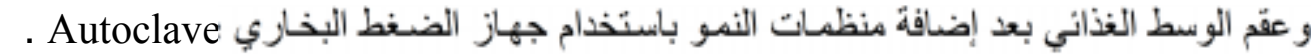
MS

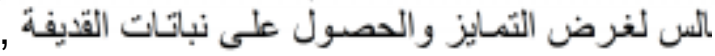
هذا الوسط لاستخدامه في زر اعة البذور

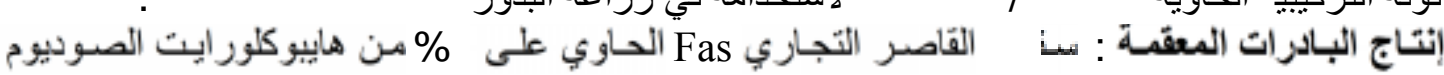

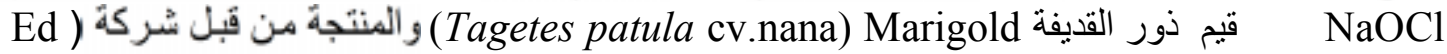
دقبقة ، تُم غسلت معقمة ثم نشرت البذور على ورق ترشيح .(Hume Seed, Inc. Puyallup. U.S.A

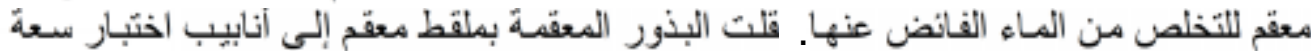

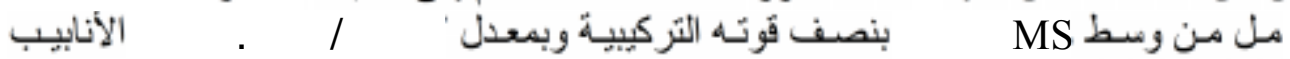

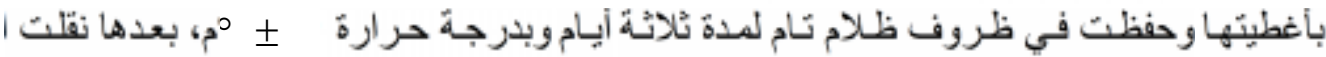

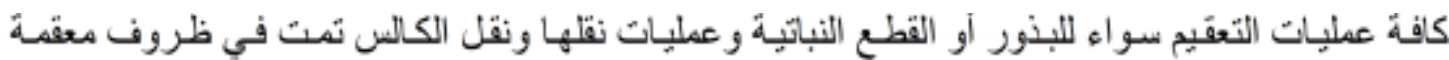

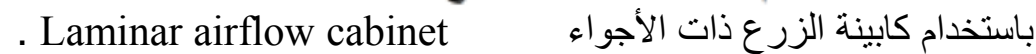

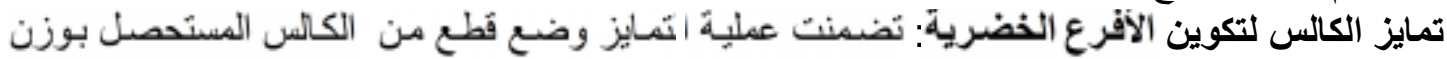

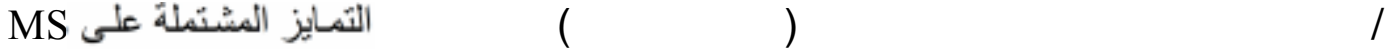

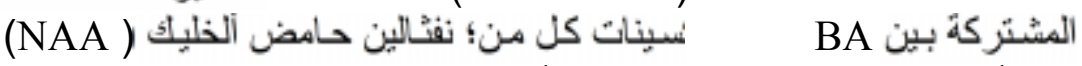
كلورو فينوكس حامض ألخليك (2,4-D ) و اندول حامض ألخليك (IAA ) بالثر اكيز التالية :

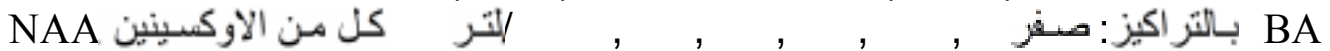

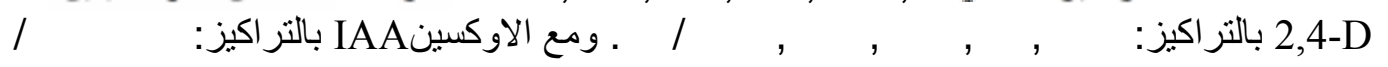
$\circ \pm$ . حفظت العينات المزروعة على هذه يومي : ليزي

الخضرية المنكونة على الكالس - أمبوع من ظهور ها

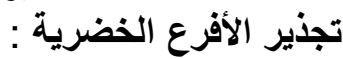
في وسط التجذير

NAA وحفظت في ظروف التحضين المُّار إليها في الفقّرة السابقة , MS

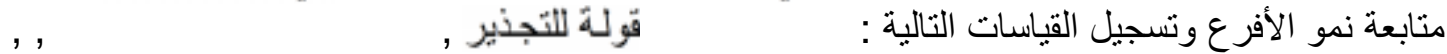

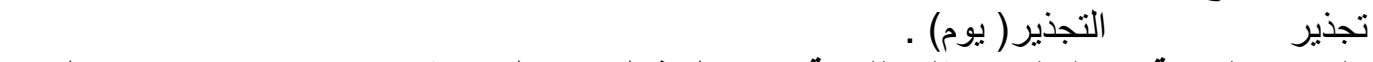

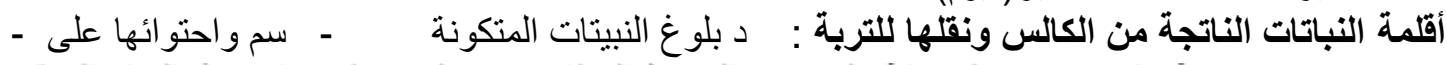

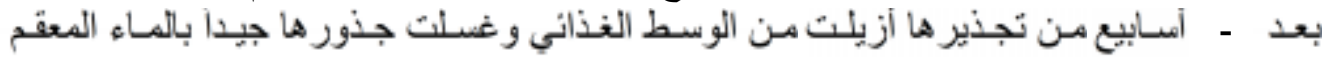

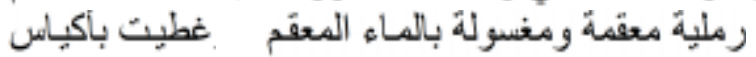

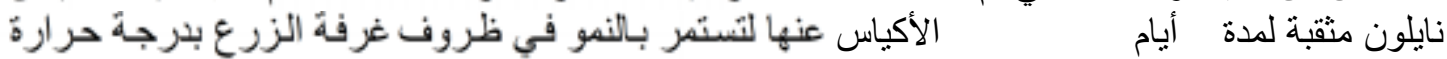

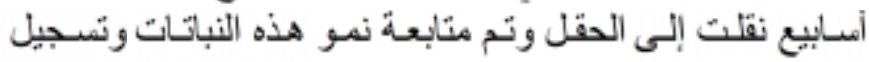

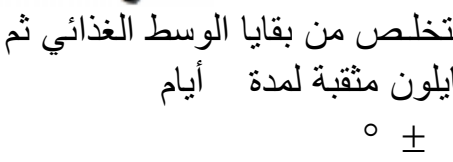
القياسات التالية : عدد النبيتات المنقولة إلى التربة ,

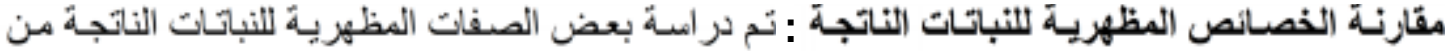

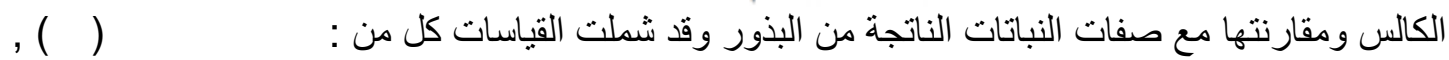

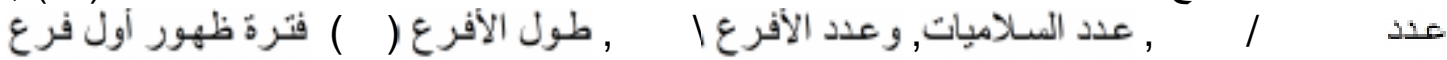

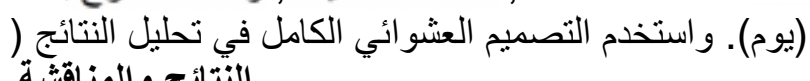
النتائج والمناقشتة

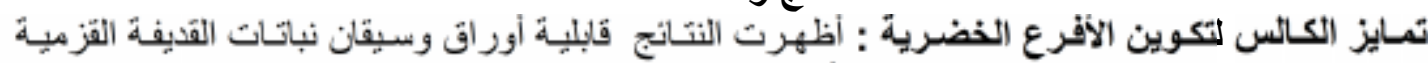
Tagetes patula cv.nana

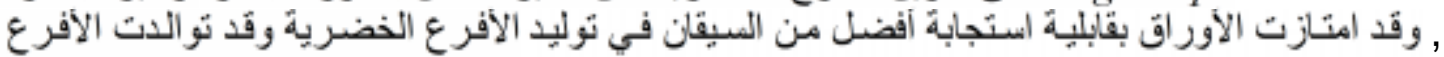

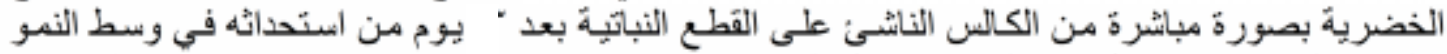

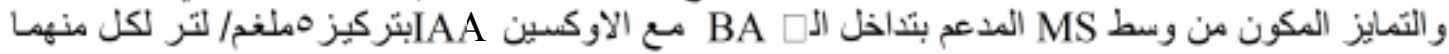
و الذي أعطى أعلى استجابة لتكوين الأفرع الخضرية بلغت الفرع لكل قُطعة من الأوراق 

( ) ( )
(ISSN 1815-316X)
مجلة زر اعة الر افدين

قطعة مستجيبة لكن هذا التذاخل لم يكن فعالا لنكوين الآفرع من قُطع

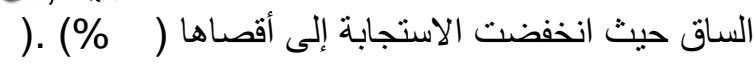

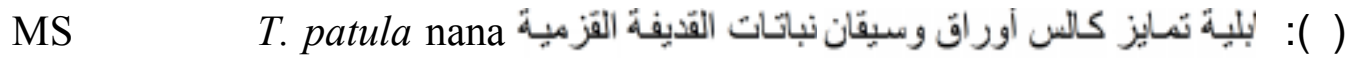

\begin{tabular}{|c|c|c|c|c|c|c|c|}
\hline مدة نشوء الأفرع ومرحلة & الأفرع / قطعة عدة & اللعأفرع الكلي & المستجيبة & اللعطدع الكلي & اللقطئ العندي & تداخط منظمات النمو في & النجاتي \\
\hline$(+\quad) 2 S R$ & & , & & & & $\mathrm{NAA},+\mathrm{BA}$ & \\
\hline , & , & , & , & , & & $2,4-\mathrm{D}, \quad+\mathrm{BA}$ & \\
\hline$(+\quad) 2 \mathrm{SR}$ & & , & & & & $2,4-\mathrm{D} \cdot+\mathrm{BA}$ & \\
\hline ( ) 1SR & & , & & & & $\mathrm{IAA}+\mathrm{BA}$ & \\
\hline$(+) 2 \mathrm{SR}$ & & $ه$ & & & & $\mathrm{NAA},+\mathrm{BA}$ & \multirow{4}{*}{ السيقان } \\
\hline$(+) 2 S R$ & & , & & & & $2,4-\mathrm{D}, \quad+\mathrm{BA}$ & \\
\hline$(+) 2 S R$ & , & , & $ه$ & & & $2,4-\mathrm{D},+\mathrm{BA}$ & \\
\hline() $1 \mathrm{SR} ه$ & & , & & $ه$ & & IAA + BA & \\
\hline , & , & , & , & , & & & \multirow{2}{*}{ 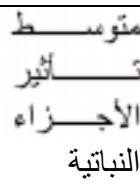 } \\
\hline , & , & , & $\cdot$ & , & & السيقان & \\
\hline
\end{tabular}

المعدلات المشتركة بنفس الأحرف في كل عمود لا تختلف معنويا حسب اختبار دنكن عند مستوى احتمالية %.

السيقان . 2SR 1SR : تعني التمايز بخطوة

الم تستجيب فيه

MS ) *

واحدة وبخطوتين One and Two Step Regeneration

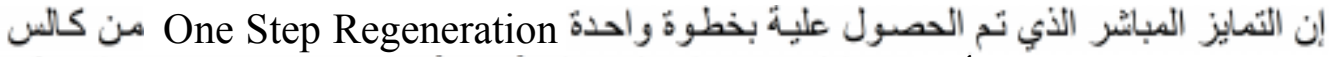

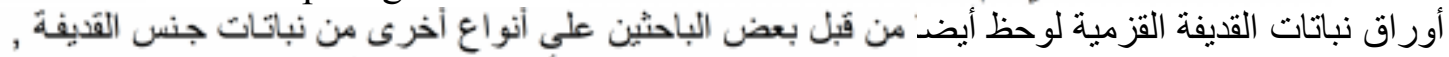
راق Datta Misra

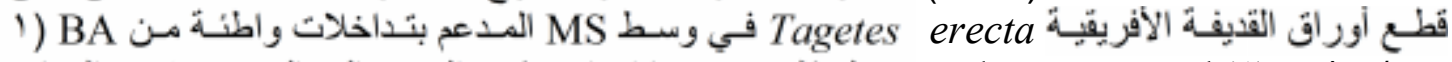

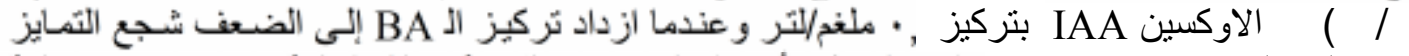

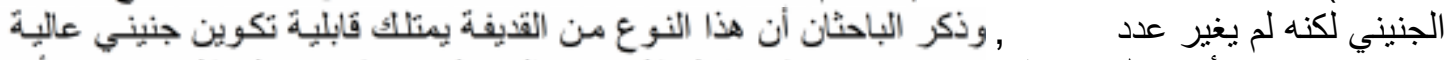

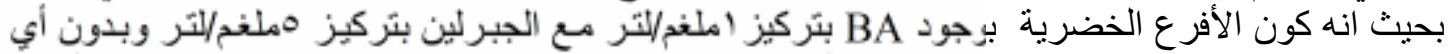

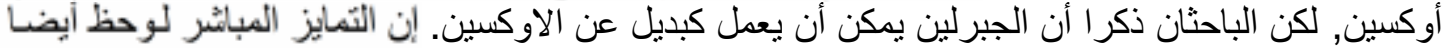

الأقحسوان Chrysanthemum مسن قبـل Chaman chamomile

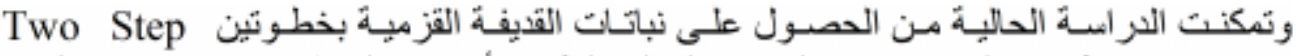

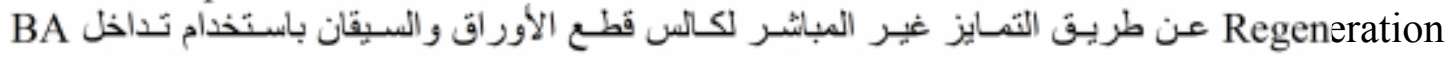

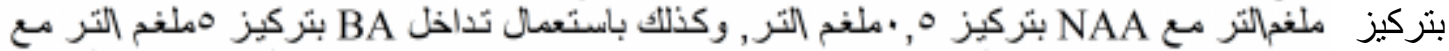

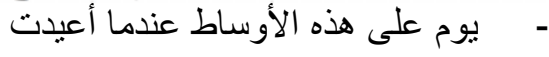
, 2,4-D / , ,

2,4-D । BA ।

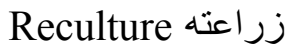

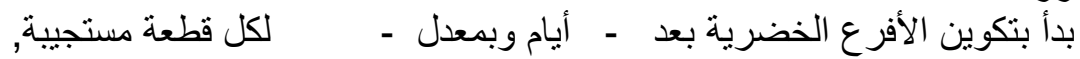

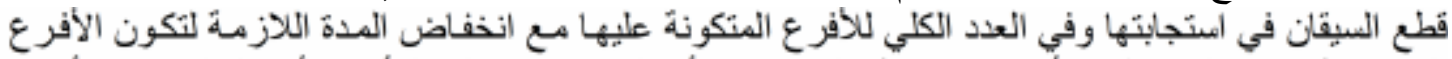

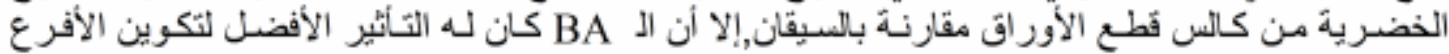

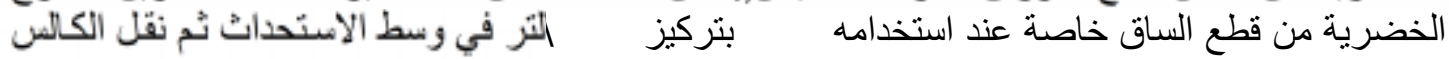

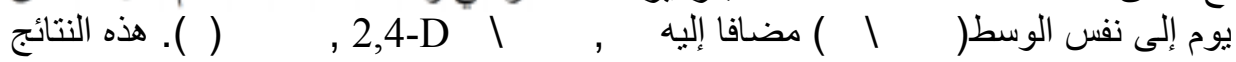




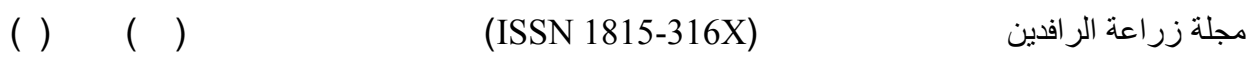

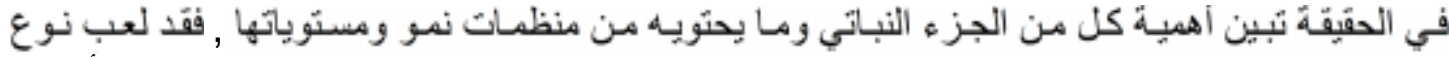

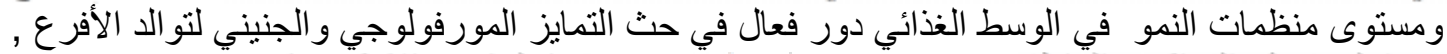

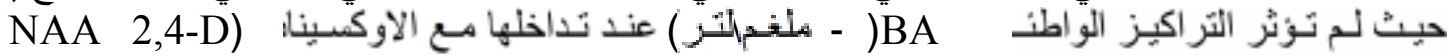

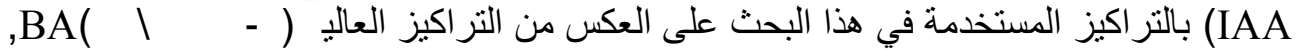

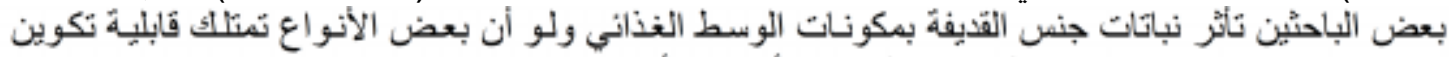

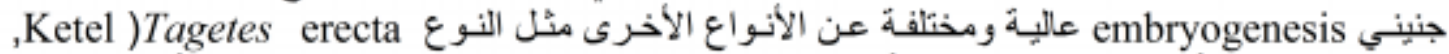

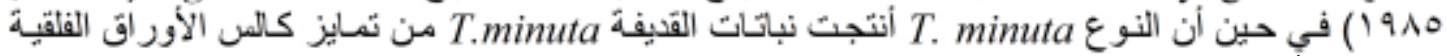

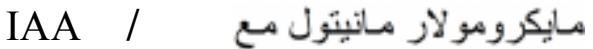

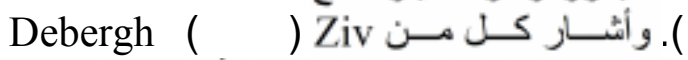

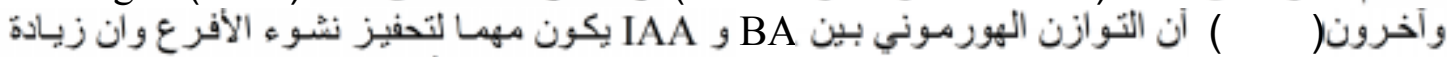

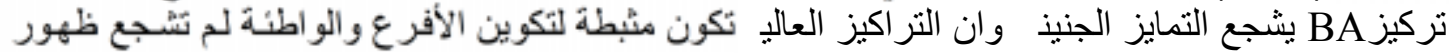

تجذير

تجذير الأفرع الخضرية : النتائج بينت قابلية الأفرع الخضرية

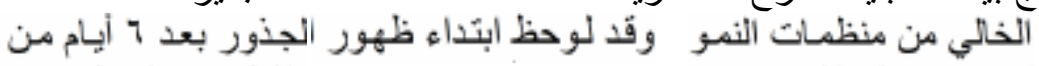

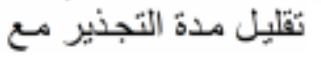
اوي •.

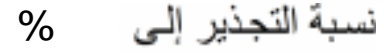

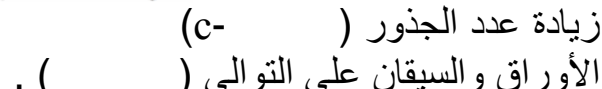

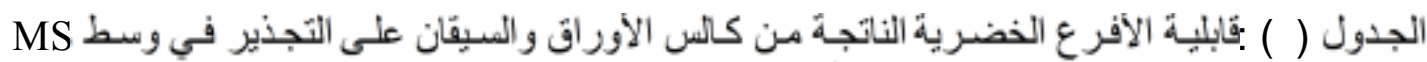

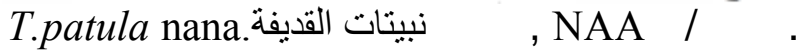

\begin{tabular}{|c|c|c|c|c|c|c|c|}
\hline 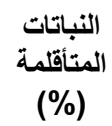 & عدد النباتات & المنقودلة إلى التيتات & 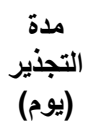 & $\begin{array}{l}\text { الأفزيرع } \\
\text { الجزير }\end{array}$ & للجذو الأفرعة & المنقولة للتجذير & المكون للأفرعاً \\
\hline & & & & $\cdot$ & & & السيقان \\
\hline
\end{tabular}

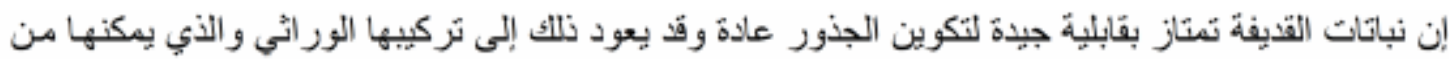

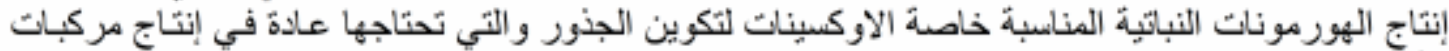

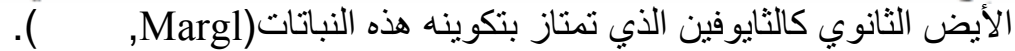

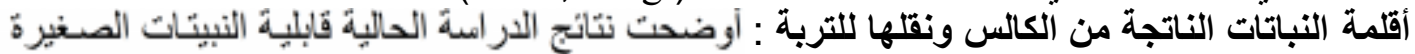

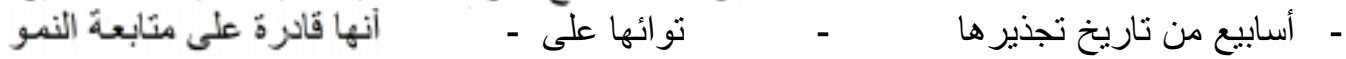

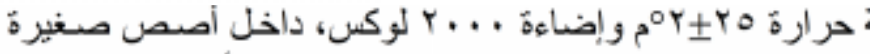

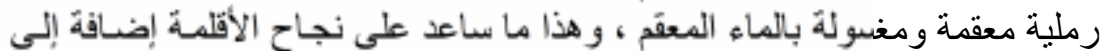

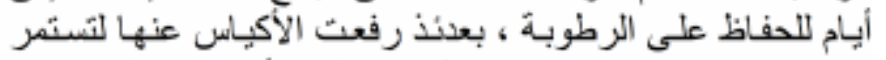

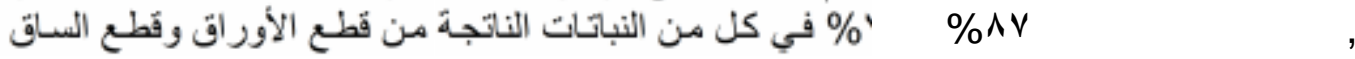

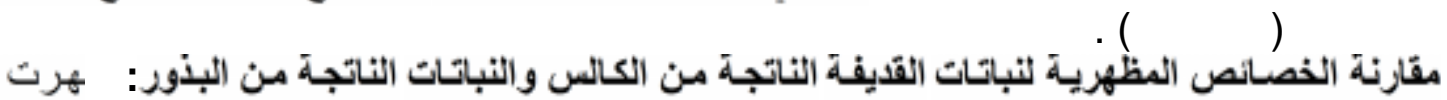

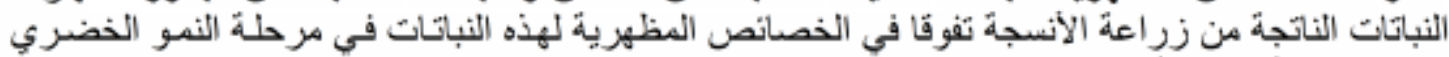

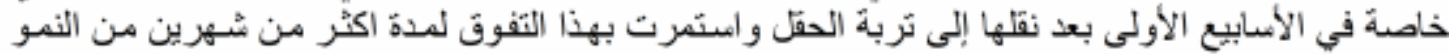

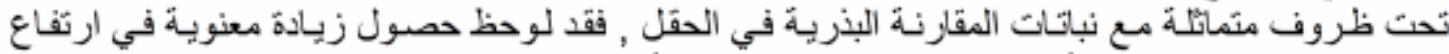

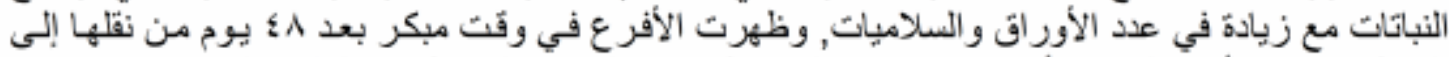

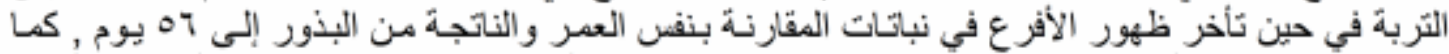

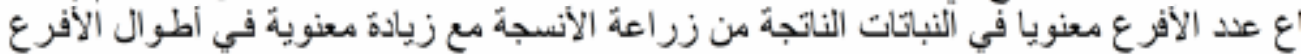



( ) ( )
(ISSN 1815-316X)
مجلة زر اعة الر افدين

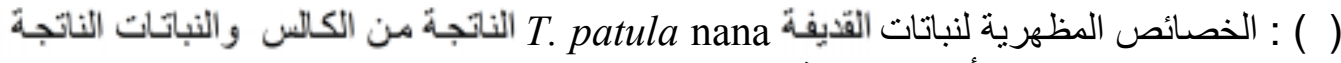

أسابيع , نامية في

\begin{tabular}{|c|c|c|c|c|c|c|}
\hline فترة ظهور أول & طول الأفرع & عدات الأفرع & عدلد سلاميات & عدد الأوراق & طول النبات & منشأ النباتات \\
\hline & & , & & & & \\
\hline & & , & & & & \\
\hline
\end{tabular}

ت المشتركة بنفس الأحرف في كل عمود لا نختلف معنويا حسب اختبار دنكن عند مستوى احتمالية %.

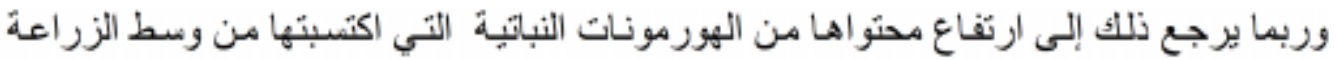

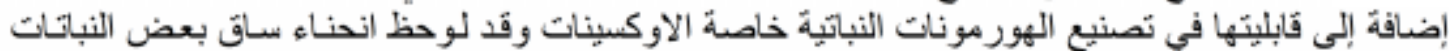

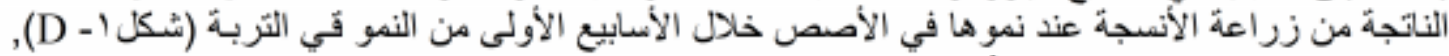

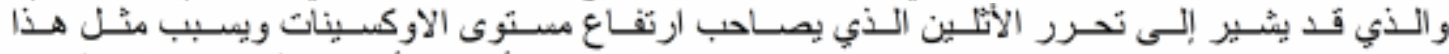

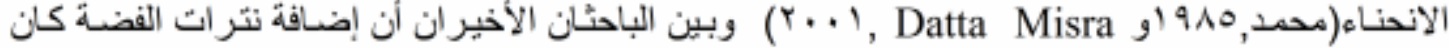

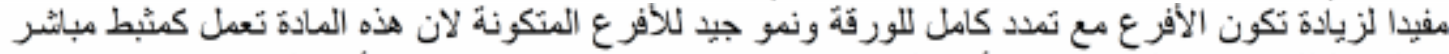

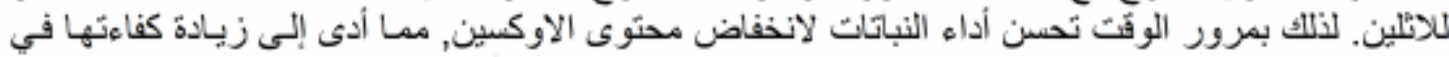

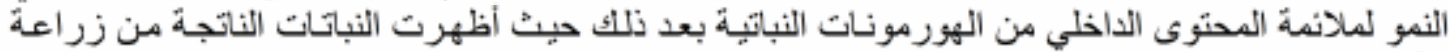

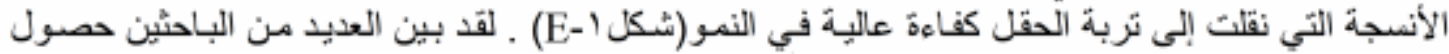

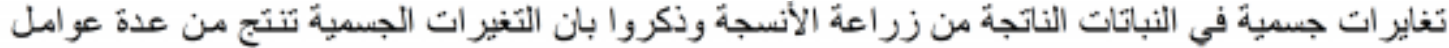

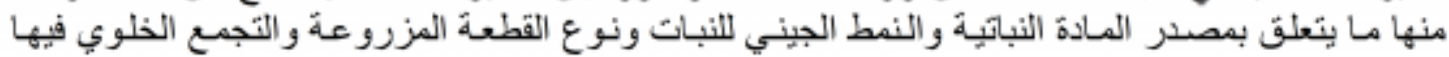

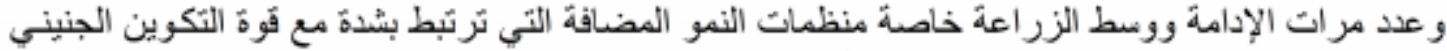
Embryogenic potential

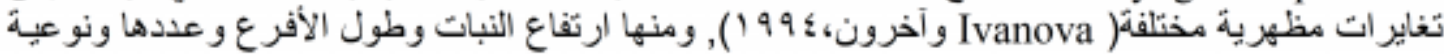

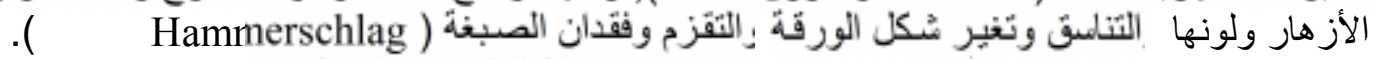

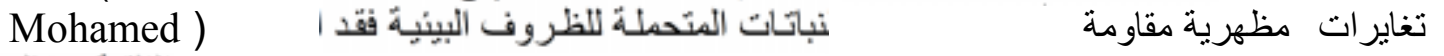

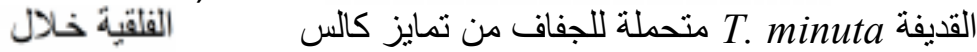

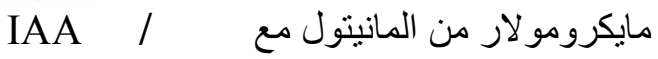

ستة اشهر من النمو في وسط BA BA / 


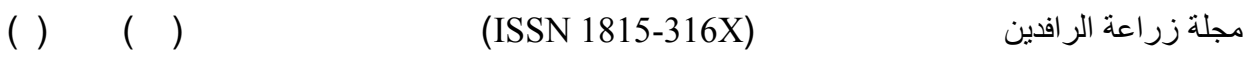
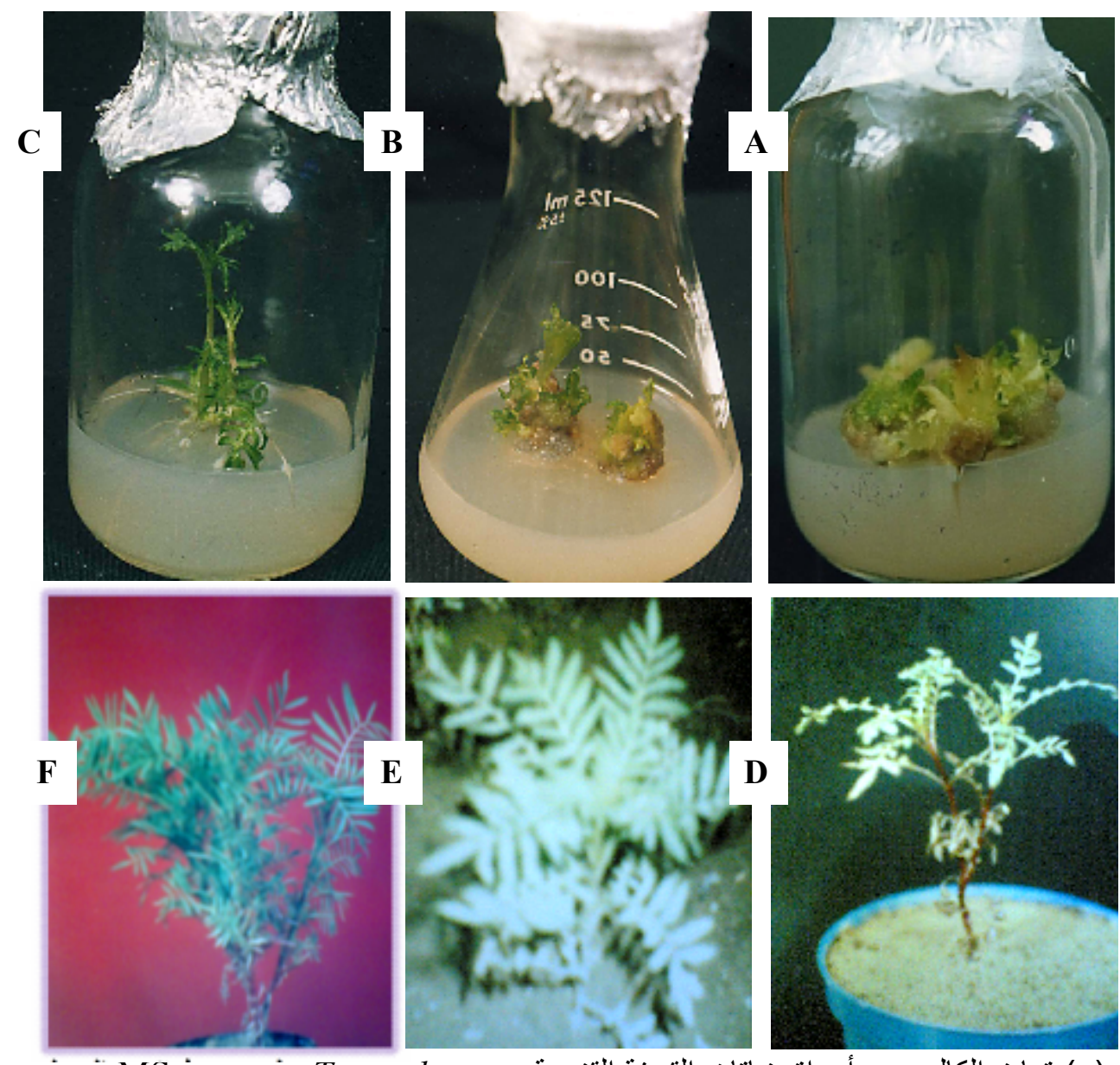

$\mathrm{MS}$

T. patula nana تمايز الكالس من أوراق نباتات القديفة القزمية ) ( )

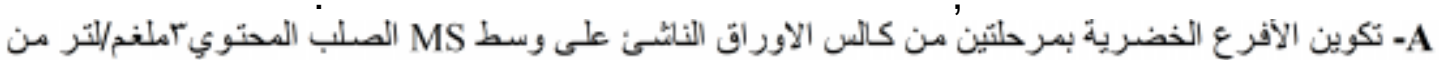

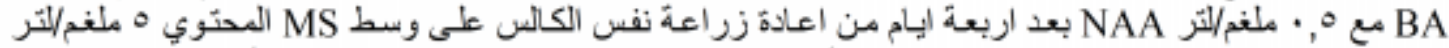

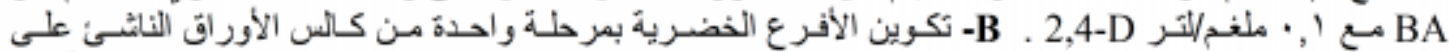

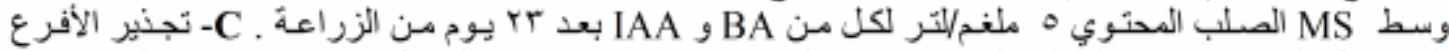

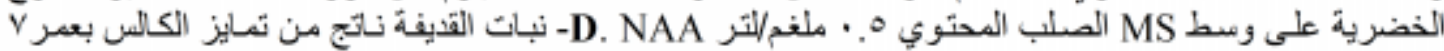

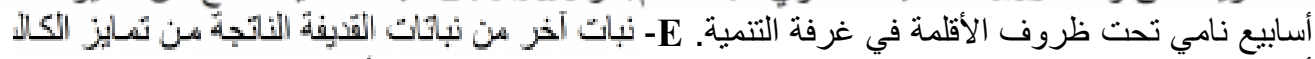
أسابيع نامي في ظروف نربة الحقل.F نبات القديفة

\section{APPLYING SOME GROWTH REGULATORS IN THE INITATION AND DIFFERENTIATION OF CALLUS TO REGENERATION OF DWARF MARIGOLD (Tagetes patula cv. nana) plants} 2- CALLUS DIFFERENTIATION AND SHOOT REGENERATION

Kasim Mahmoud Al-Hamdani

Dept. of Crop/ College of Agric. and Forestry, Univ. of Mosul, Iraq

\section{ABSTRACT}

This study showed the differentiation capability of dwarf Tagetes patula cv. nana callus to regenerated of plants directly and indirectly on in vitro aseptic medium, whereas was obtained of shoot organogenesis by two methods. The first by direct differentiation of callus which initiated on solidified MS medium 


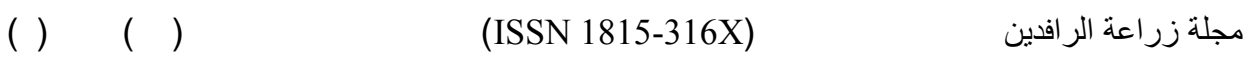

containing $5 \mathrm{mgL}^{-1}$ of each of BA and IAA after 23 days of culture ,the second method was from indirect differentiation of callus on MS medium supplemented with $3 \mathrm{mgL}^{-1}$ BA with $0.5 \mathrm{mgL}^{-1} \mathrm{NAA}$. The shoot arise after four days of transplanting of callus (21 days age) to same medium or to MS medium containing $5 \mathrm{mgL}^{-1}$ of BA with $0.1 \mathrm{mgL}^{-1} 2,4-\mathrm{D}$. The producing shoots were readily rooted on MS medium containing $0.5 \mathrm{mgL}^{-1}$ of NAA. , and subsequently the plantlet producing from leaves callus were adapted(77.8\%) to the environmental conditions through transplanting them in soil. they were showed faster growth in the first vegetative growth stage compared with seed plants.

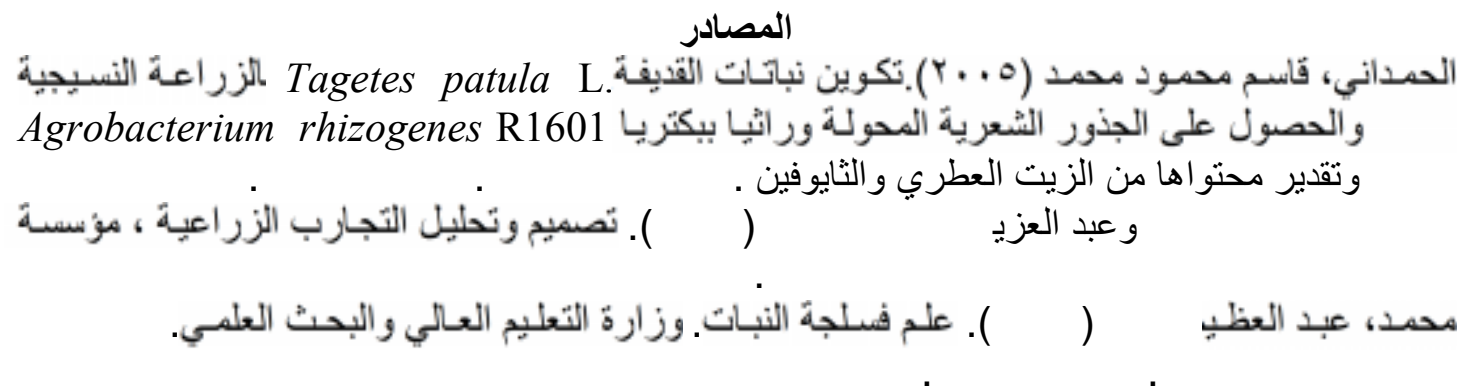

Breteler,H , CH. H. Hanisch ten Cate. and P. Nissen (1979). Time-course of nitrate uptake and nitrate reductase activity in nitrogen-depleted dwarf bean. Physiol. Plant. 47:49-55.

Chakrabarty, D., A. K. A. Mandal. and S. K. Datta (2000). Retrieval of new coloured chrysanthemum through organogenesis from sectorial chimera. Curr.Sci. 78:1060-1061 .

Debergh, P., J. Aitken-Christie., D. Cohen., B. Grout., S. Von Arnold., R. Zimmerman., and M. Ziv (1992). Reconsideration of the term vitrification as used in micropropagation. Plant Cell Tiss. Organ Cult. 30:135-140.

Echeverrigaray, S., F. Fracaro., L.B. Andrade., S. Biasio., and L. Atti-Seratini (2000). In vitro shoot regeneration from leaf explants of Roman Chamomile.Plant Cell Tiss. Organ Cult. 60:1-4.

Hammerschlag, F.A. (1992). Somaclonal variation. In: Hammerschlag, F.A. and Litz, R.E. (eds.). Biotechnology of Perennial Fruit Crops. C.A.B. International. U.K. PP. 35-56.

Ivanova, A., M. Velcheva., P.Denchev., A. Atanssov, and A. Van. Onklelen (1994). Endogenous hormone levels during direct somatic embryogenesis in Medicago spp. Physiol. Plant. 92:85-89.

Ketel, D.H. (1986).Morphological differentiation and occurrence of thiophenes in leaf callus cultures from Tagetes species: Relation to the growth medium of the plants. Physiol. Plant. 66:392-396.

Ketel, D.H., H. Breteler., and B. de Groot (1985). Effect of explant origin on growth and differentiation of calli from Tagetes species. J. plant physiol. 118:327-333.

Margl, L., A. Tei., I. Gyurjan., and M.Wink (2002). GLC and GLC-MS analysis of thiophene derivatives in plants and in In vitro cultures of Tagetes patula (Asteraceae).Z. Naturforsch. 57: 63-71. 


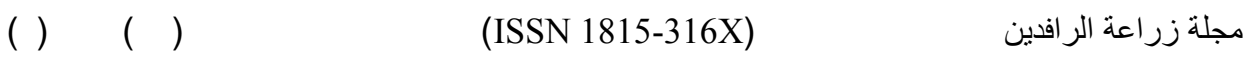

Misra, P. and S.K. Datta (2001). Direct differentiation of shoot buds in leaf segments of white marigold (Tagetes erecta L.). In vitro Cell. Dev. Biol.Plant. 37:466-470.

Mohamed, M.A.H., P.J.C. Harris., and J. Henderson (2000). In vitro selection and characterisation of a drought tolerant clone of Tagetes minuta. Plant Sci. 159:213-222.

Murashige, T. and F. Skoog ( 1962). A revised medium for rapid growth and bioassays with tobacco tissue cultures. Physiol. Plant. 15:473-479.

Ziv, M. (1991). Vitrification morphological and physiological disorders of in vitro plants. In: Debergh, P. C.; Zimmerman, R. H., eds. Micropropagation \pm technology and application. Dordrecht: Kluwer Academic Publishers; 45-69. 